\title{
Frequency and spectrum of PIK3CA somatic mutations in breast cancer
}

\author{
Olga Martínez-Sáez ${ }^{1,2,3}$, Nuria Chic ${ }^{1,2,3}$, Tomás Pascual ${ }^{1,2,3}$, Barbara Adamo ${ }^{1,2,3}$, Maria Vidal ${ }^{1,2,3}$, \\ Blanca González-Farré 1,2,3,4, Esther Sanfeliu ${ }^{1,2,3,4}$, Francesco Schettini ${ }^{1,2,3,5}$, Benedetta Conte ${ }^{1,2,3}$, \\ Fara Brasó-Maristany 1,2,3, Adela Rodríguez¹, Débora Martínez², Patricia Galván², Ana Belén Rodríguez', \\ Antonio Martinez ${ }^{5}$, Montserrat Muñoz ${ }^{1,2,3}$ and Aleix Prat ${ }^{1,2,3^{*}}$
}

\begin{abstract}
Purpose: The therascreen PIK3CA mutation assay and the alpha-specific PI3K inhibitor alpelisib are FDA-approved for identifying and treating patients with advanced PIK3CA-mutated (PIK3CAmut) breast cancer (BC). However, it is currently unknown to what extend this assay detects most PIK3CA mutations in BC. This information is critical as patients and clinicians are using this and other genomic assays to indicate alpelisib.
\end{abstract}

Methods: Data from 6338 patients with BC was explored across 10 publicly available studies. The primary objective was to evaluate the proportion and distribution of PIK3CA mutations in BC. Secondary objectives were (1) to evaluate in silico the spectrum of PIK3CA mutations in BC that would be captured by the therascreen panel; (2) to evaluate the proportion and distribution of PIK3CA mutations in hormone receptor-positive/HER2-negative (HR+/ HER2-), HER2+, and triple-negative BC (TNBC); and (3) to explore the identification of PIK3CA mutations in a cohort of 48 HR+/HER2 - advanced BC patients by the Guardant B360 circulating tumor DNA (ctDNA) assay.

Results: Patients with PIK3CAmut tumors represented 35.7\% (2261/6338). Five PIK3CA mutations comprised 73\% of all PIK3CA mutations: H1047R (35\%), E545K (17\%), E542K (11\%), N345K (6\%), and H1047L (4\%). Therascreen gene list would capture $72 \%$ of all PIK3CA mutations and $80 \%$ of patients with a known PIK3CAmut BC. Among patients with double PIK3CAmut tumors (12\% of all PIK3CAmut), the therascreen panel would capture $78 \%$ as harboring 1 single PIK3CA mutation, 17\% as PIK3CAmut undetected, and 5\% as PIK3CA double-mut. PIK3CA mutation rates were lower in TNBC (16\%) compared to HR+/HER2 (42\%) and HER2+ (31\%) BC; however, the distribution of the 4 main PIK3CA mutations across subtypes was similar. Finally, 28\% of PIK3CA mutations identified in ctDNA in 48 patients with advanced HR+/HER2- BC were not part of the therascreen panel.

(Continued on next page)

\footnotetext{
* Correspondence: alprat@clinic.cat

'Department of Medical Oncology, Hospital Clinic of Barcelona, Villarroel 170, 08035 Barcelona, Spain

${ }^{2}$ Translational Genomics and Targeted Therapies in Solid Tumors, Institut D'Investigacions Biomèdiques August Pi i Sunyer (IDIBAPS), Villarroel 170, 08035 Barcelona, Spain

Full list of author information is available at the end of the article
}

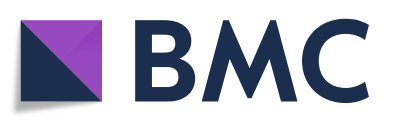

(c) The Author(s). 2020 Open Access This article is licensed under a Creative Commons Attribution 4.0 International License, which permits use, sharing, adaptation, distribution and reproduction in any medium or format, as long as you give appropriate credit to the original author(s) and the source, provide a link to the Creative Commons licence, and indicate if changes were made. The images or other third party material in this article are included in the article's Creative Commons licence, unless indicated otherwise in a credit line to the material. If material is not included in the article's Creative Commons licence and your intended use is not permitted by statutory regulation or exceeds the permitted use, you will need to obtain permission directly from the copyright holder. To view a copy of this licence, visit http://creativecommons.org/licenses/by/4.0/ The Creative Commons Public Domain Dedication waiver (http://creativecommons.org/publicdomain/zero/1.0/) applies to the data made available in this article, unless otherwise stated in a credit line to the data. 


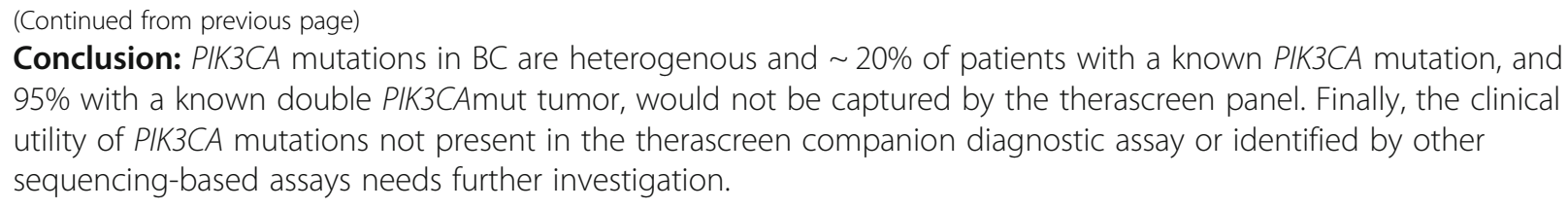

Keywords: Breast cancer, PIK3CA, Mutations, Alpelisib, Companion diagnostic, Hotspot mutations, Therascreen, ctDNA

\section{Introduction}

Activating mutations in the PIK3CA are found in approximately $30-40 \%$ of patients with cancer and induce

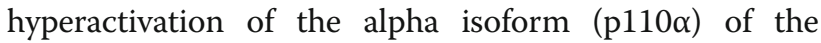
phosphatidylinositol 3-kinase (PI3K) [1-3]. In patients with $\mathrm{HR}+/$ HER2 - BC, mTOR/mTOR pathway has been associated with endocrine therapy resistance [4]. In addition, the role of this pathway is becoming increasingly important in HER2+ and TNBC [5-7]. Thus, inhibition of PI3K in PIK3CA-mutated $\mathrm{BC}$ has been a major focus in the last decade [3].

Alpelisib is an orally bioavailable, small-molecule, $\alpha$ specific PI3K inhibitor that inhibits $\mathrm{p} 110 \alpha$ approximately 50 times as strongly as other isoforms [8]. Following successful preclinical and phase 1 data $[4,9]$, the SOLAR-1 phase III randomized trial evaluated the efficacy of alpelisib plus fulvestrant in 572 patients with HR+/HER2 - advanced BC who had received prior endocrine therapy [10]. A clinically relevant treatment benefit was only observed in the cohort of patients with PIK3CAmut disease. In May 2019, the FDA approved alpelisib for the treatment of patients with advanced PIK3CAmut HR+/HER2- BC.

Together with alpelisib, the FDA also approved the companion diagnostic therascreen ${ }^{\bullet}$ PIK3CA test (QIAGEN Manchester, Ltd.) used in SOLAR-1 to select patients who had PIK3CA mutations in tumor tissue specimens and/or in circulating tumor DNA (ctDNA) isolated from plasma specimens [11]. Therascreen PIK3CA detects 11 PIK3CA hotspot mutations, mostly found in exons 9 and 20 [11]. In SOLAR-1, the type of PIK3CA mutation did not seem to impact the main results [10].

In this context, patients and physicians might choose not to use the therascreen PIK3CA test and use other available tests, which provide a more comprehensive mutational analysis of PIK3CA as well as other genes. This might lead to the clinical situation where PIK3CA mutations not detected by the therascreen PIK3CA assay, and thus not evaluated in SOLAR-1, are used to indicate alpelisib. To define the potential frequency of this clinical situation, here we aimed to evaluate the distribution of PIK3CA mutations in $\mathrm{BC}$ in relation to the therascreen PIK3CA panel.

\section{Methods}

\section{Datasets}

All non-overlapping publicly available breast datasets (i.e., 12 studies and 6477 samples) (https://www.mbcproject.org/data-release [1,12-21]) with PIK3CA mutational status were interrogated from cBio Cancer Genomics Portal (http://cbioportal.org) [22] (Fig. 1 and Additional file 1). Among them, 2 studies focused on 117 patient-derived xenografts [16] and 22 fibroepithelial lesions of the breast [17] were removed. The remaining combined dataset included 6338 invasive tumor samples of which 5535 (87.3\%) originated from the METABRIC $(n=2509)$, the Memorial Sloan-Kettering $(n=1918)$, and The Cancer Genome Atlas $(n=1108)$ datasets. All studies analyzed performed targeted or whole exome sequencing (Table A1 Additional file 1). Only single nucleotide variants, insertions, or short deletions in PIK3CA were analyzed.

\section{Therascreen PIK3CA mutation assay}

The therascreen ${ }^{\circ}$ PIK3CA RGQ PCR Kit is a real-time qualitative PCR test for the detection of 11 mutations in PIK3CA gene (exon 7: C420R; exon 9: E542K, E545A, E545D, E545G, E545K, Q546E, and Q546R; and exon 20: H1047L, H1047R, and H1047Y) using genomic DNA extracted from formalin-fixed, paraffin-embedded breast tumor tissue or ctDNA from plasma derived from K2EDTA anticoagulated peripheral whole blood taken from patients with $\mathrm{BC}$ [11].

\section{Immunohistochemistry (IHC)-based subtypes}

IHC data was available from 5813 patients (92\%). Tumors were classified into the 3 main clinically relevant subtypes: (1) HR+/HER2-, (2) HER2+, and (3) TNBC. Tumors identified as progesterone receptor positive and HER2- were considered HR + regardless of estrogen receptor (ER) status. Tumors identified as ER-negative and HER2- were considered TNBC when progesterone receptor status was not available.

\section{Distribution of PIK3CA mutations in plasma}

Frozen plasma samples from 48 patients with advanced HR+/HER2- BC were obtained before initiating a CDK4/ 6 inhibitor and endocrine therapy. Plasma samples were 


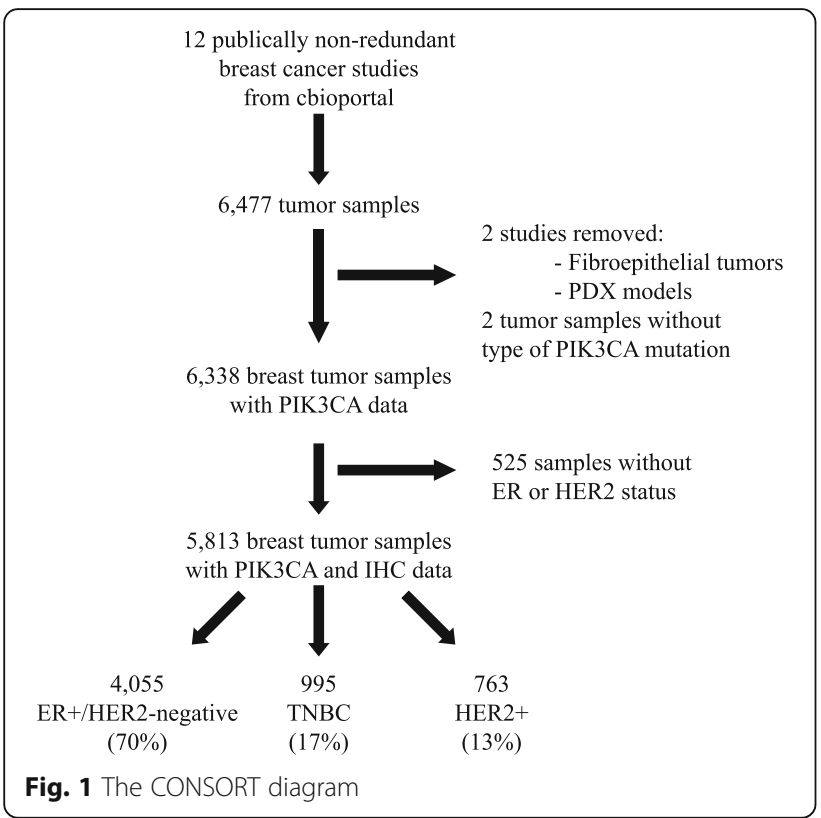

sent to Guardant Health (California, USA), and the 74gene standardized NGS-based assay, which includes all 21 exons from the PIK3CA gene, was performed.

\section{Study end points}

Primary objective was to evaluate the proportion and distribution of PIK3CA mutations in BC. Secondary objectives were (1) to evaluate in silico the spectrum of PIK3CA mutations in $\mathrm{BC}$ that would be captured by the therascreen panel; (2) to evaluate the proportion and distribution of PIK3CA mutations in HR+/HER2-, HER2+, and TNBC; and (3) to explore the identification of PIK3CA mutations in HR+/HER2- advanced BC by the Guardant B360 ctDNA assay.

\section{Statistical analyses}

Patient and tumor characteristics were analyzed using descriptive statistics.

\section{A}

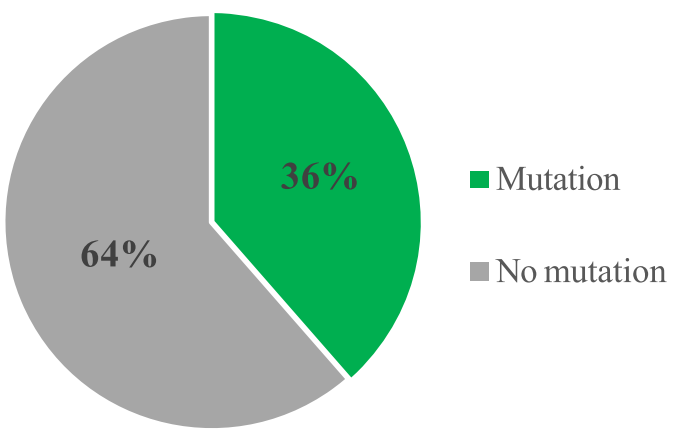

$\mathbf{C}$

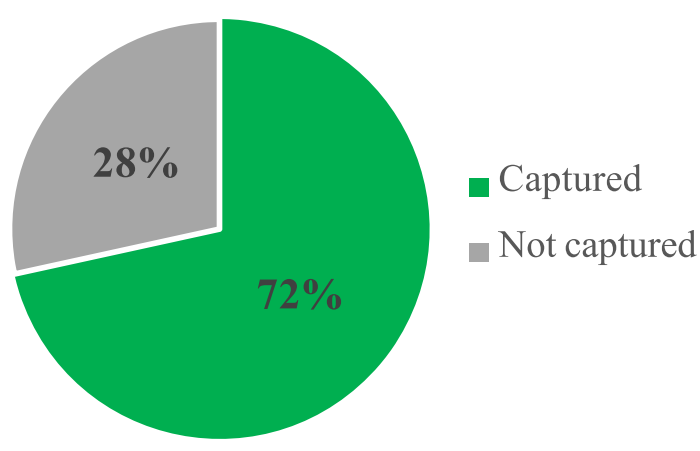

\section{B}

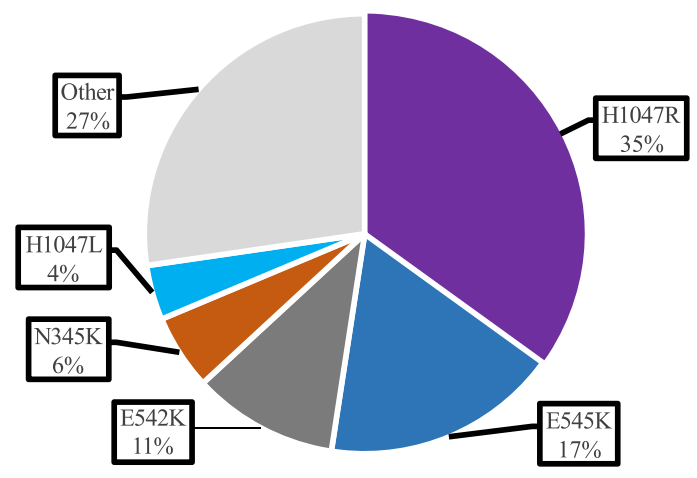

D

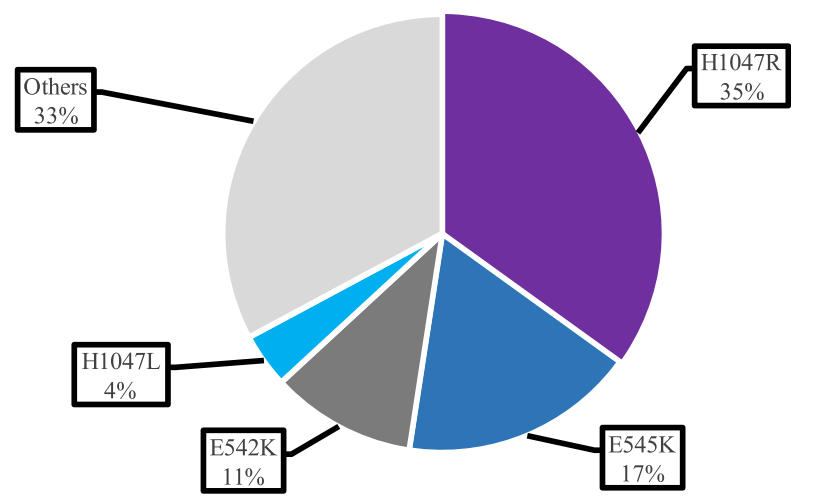

Fig. 2 Proportion of PIK3CA mutations in $B C$ in relation to the mutations detected by therascreen. a Proportion of patients with PIK3CA mutations in the combined dataset. $\mathbf{b}$ Distribution of the various types of PIK3CA mutations in the combined dataset. $\mathbf{c}$ Proportion of PIK3CA mutations detected by the therascreen assay. $\mathbf{d}$ Distribution of the various types of PIK3CA mutations detected by the therascreen assay in the combined dataset 
Table 1 The 20 most frequent PIK3CA mutations in BC

\begin{tabular}{|c|c|c|c|c|c|c|}
\hline $\begin{array}{l}\text { Type of PIK3CA } \\
\text { mutation }\end{array}$ & Exon & $\begin{array}{l}\text { Oncogenic by } \\
\text { OncoKB }{ }^{24}\end{array}$ & $\begin{array}{l}\text { Level of evidence to } \\
\text { predict alpelisib benefit }\end{array}$ & $\begin{array}{l}\text { Detected by } \\
\text { therascreen }\end{array}$ & $\begin{array}{l}\text { Number of mutations found } \\
\text { in the combined dataset }\end{array}$ & $\begin{array}{l}\text { Mutation } \\
\text { frequency (\%) }\end{array}$ \\
\hline H1047R & 20 & Yes & 1 & Yes & 895 & 35.0 \\
\hline E545K & 9 & Yes & 1 & Yes & 447 & 17.5 \\
\hline E542K & 9 & Yes & 1 & Yes & 274 & 10.7 \\
\hline N345K & 4 & Yes & Yes (preclinical only) & No & 142 & 5.5 \\
\hline H1047L & 20 & Yes & 1 & Yes & 103 & 4.0 \\
\hline E726K & 13 & $\begin{array}{l}\text { Inconclusive. } \\
\text { Probably oncogenic }\end{array}$ & Unknown & No & 65 & 2.5 \\
\hline C420R & 7 & Yes & 1 & Yes & 48 & 1.9 \\
\hline Q546R & 9 & Yes & 1 & Yes & 27 & 1.1 \\
\hline G118D & 1 & Yes & Unknown & No & 26 & 1.0 \\
\hline E453K & 7 & Yes & Unknown & No & 22 & 0.9 \\
\hline Q546K & 1 & Yes & Yes (preclinical only) & No & 21 & 0.8 \\
\hline G1049R & 20 & Yes & Yes (preclinical only) & No & 19 & 0.7 \\
\hline M1043I & 20 & Yes & Unknown & No & 19 & 0.7 \\
\hline $\mathrm{K} 111 \mathrm{E}$ & 1 & Yes & Unknown & No & 16 & 0.6 \\
\hline E81K & 1 & $\begin{array}{l}\text { Inconclusive. } \\
\text { Probably oncogenic }\end{array}$ & Unknown & No & 15 & 0.6 \\
\hline E545A & 9 & Yes & 1 & Yes & 13 & 0.5 \\
\hline E545G & 9 & Yes & 1 & Yes & 13 & 0.5 \\
\hline N1044K & 20 & Yes & Unknown & No & 12 & 0.5 \\
\hline E110del & 1 & Yes & Unknown & No & 11 & 0.4 \\
\hline Q546P & 9 & Yes & Unknown & No & 10 & 0.4 \\
\hline
\end{tabular}

\section{Results}

\section{Distribution of PIK3CA mutations in BC}

In the combined dataset, $36 \%$ of patients had PIK3$C A$ mut tumors (Fig. 2a). From a total of 2560 PIK3CA mutations, 205 PIK3CA mutations were unique. The most frequent PIK3CA mutations (i.e., frequency $\geq 4 \%$ of all PIK3CAmut tumors) were found in exons 4, 9, and 20: H1047R (35\%), E545K (17\%), E542K (11\%), N345K (6\%), and H1047L (4\%) (Table 1, Fig. 2b). These 5 mutations comprised $73 \%$ of all PIK3CA mutations identified in the combined dataset.

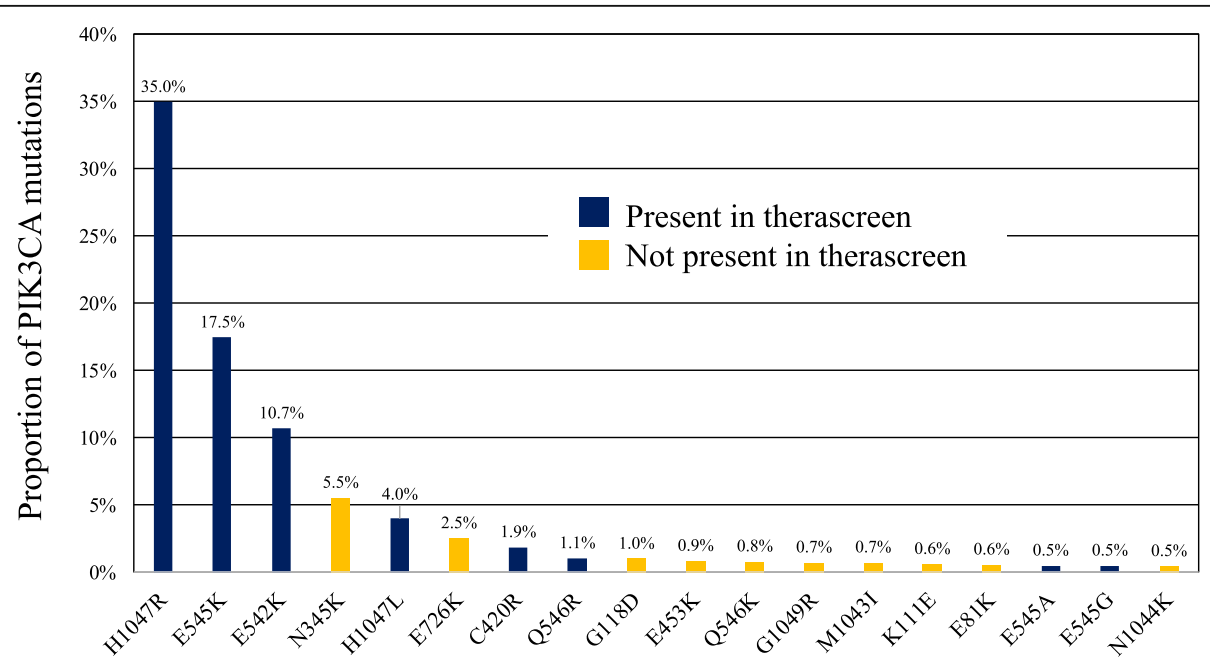

Fig. 3 Proportion of the 18 most frequent PIK3CA mutations in PIK3CAmut BC in the combined dataset 
PIK3CA mutations captured by the therascreen panel In the combined dataset, the proportion of PIK3CA mutations included in the therascreen panel was $72 \%$ (Fig. 2c). The most frequent types of PIK3CA mutation (i.e., frequency $\geq 4 \%$ of all PIK3CAmut tumors) included in the therascreen panel were H1047R (35\%), E545K (17\%), E542K (11\%), and H1047L (4\%) (Fig. 2d). These 4 mutations comprised $67 \%$ of all PIK3CA mutations detected in the dataset. Of note, N345K mutation in exon 4, which represents $6 \%$ of all tumor samples with a PIK3CA mutation, is not part of the therascreen panel (Fig. 3). Although the clinical utility of non-therascreen-detected PIK3CA mutations is currently unknown, the N345K lies within the C2 PI3K-type domain of the protein and confers a gain of function on PI3K, as does C420R (a tested mutation by therascreen assay) [23]. Moreover, N345K mutation has shown increased sensitivity to PI3K pathway inhibition in preclinical models [24]. Interestingly, Q546E PIK3CA mutation included in the therascreen panel was not found in the combined dataset.

\section{Detection of multiple PIK3CA mutations in a tumor sample}

Among 2261 patients with PIK3CAmut tumors, 1979 (87.5\%) had 1 single mutation, 267 (11.8\%) had 2 mutations, and $15(0.7 \%)$ had 3 or more mutations. Overall, patients with tumors harboring double PIK3CA mutations represented $4 \%$ of all BC (Fig. 4a). Among patients with 1 single PIK3CA mutation, $80 \%$ would have mutations represented in the therascreen mutational panel (Fig. 4b). Among patients with 2 or more PIK3CA mutations, $78 \%$ would have 1 mutation represented in the therascreen panel; $17 \%$, no mutation represented in the therascreen panel; and 5\%, 2 or more mutations represented in the therascreen panel (Fig. 4c).

\section{PIK3CA mutational distribution according to subtypes in the $B C$ dataset}

Among 5813 patients with IHC data, 4055 (70\%) had HR+/HER2- disease, 995 had TNBC (17\%), and 763
(13\%) had HER2+ disease. PIK3CA mutations were less frequent in TNBC (16\%) than in HR+/HER2- (42\%) or HER2+ disease (31\%) (Fig. 5a-c). However, the distribution of PIK3CA mutations was similar across subtypes (Fig. 5d-f). Seventy-one percent of mutations in $\mathrm{HR}+/$ HER2- BC, 75\% in HER2+ BC, and 72\% in TNBC would be represented in the therascreen panel.

\section{Distribution of PIK3CA mutations in plasma ctDNA}

Therascreen assay is approved for detecting PIK3CA mutations in ctDNA from plasma samples [25]. To evaluate the distribution of $P I K 3 C A$ mutations in ctDNA using a highly sensitive assay that sequences all 21 PIK3CA exons, we tested plasma samples from 48 consecutive patients with metastatic $\mathrm{HR}+$ /HER2- BC from the Hospital Clinic of Barcelona using the Guardant B360 standardized assay [26]. All patients had recurred or progressed to prior lines and were about to initiate a CDK4/6 inhibitor and endocrine therapy. A PIK3CA mutation was detected in 17 patients (37\%), and 1 patient (6\%) had double PIK3CA mutation. The spectrum of PIK3CA mutations was similar to the one found in the previous in silico population analysis (Table A2 Additional file 1). More importantly, 5 patients (28\%) had PIK3CA mutations not represented in the therascreen mutational panel.

\section{Discussion}

PIK3CA mutations have recently reached level 1 evidence for predicting benefit from alpelisib, an alphaspecific PI3K inhibitor, in combination with fulvestrant in patients with advanced HR+/HER2- BC previously treated with endocrine therapy [10]. In addition, several trials are now evaluating alpelisib and other alphaspecific PI3K inhibitors in other $\mathrm{BC}$ subtypes harboring PIK3CA mutations [27]. Thus, there is a need to better understand the heterogeneity of the mutational landscape of PIK3CA and, at the same time, relate this heterogeneity with the recently introduced therascreen

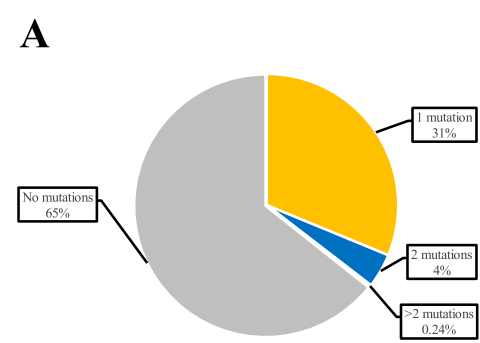

B

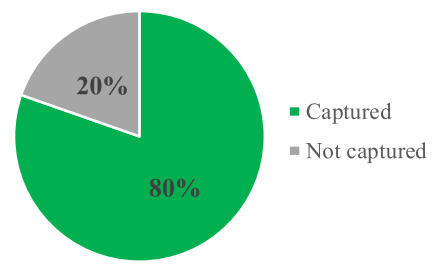

C

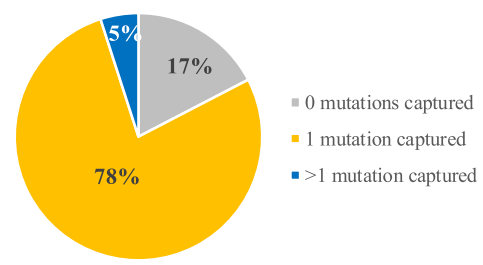

Fig. 4 Proportion of patients with PIK3CA mutations in $B C$ in relation to the mutations detected by therascreen. a Proportion of patients with one, two, or three or more PIK3CA mutations in BC in the combined dataset. $\mathbf{b}$ Proportion of patients with a single PIK3CA mutation detected PIK3CAmut by the therascreen assay. $\mathbf{c}$ Proportion of patients with two or more PIK3CA mutations detected by the therascreen assay as either PIK3CA mutation "not detected," single PIK3CA mutation or as harboring 2 or more PIK3CA mutations 


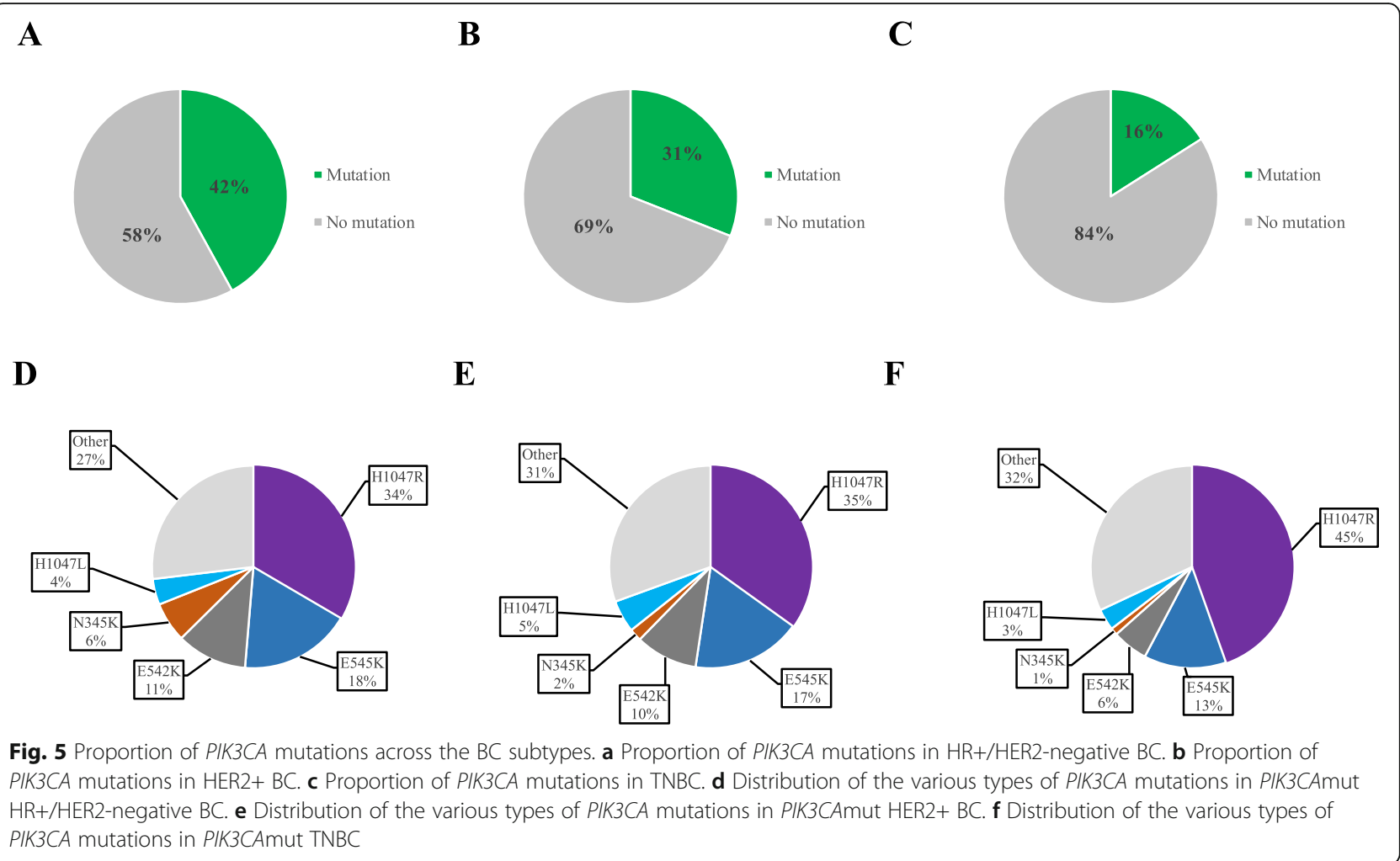

PIK3CA companion diagnostic assay approved to indicate alpelisib.

To address this topic, we performed a comprehensive evaluation of the distribution of PIK3CA mutations in $\mathrm{BC}$ and made the following observations. First, although PIK3CA mutations are highly heterogeneous, 5 mutations (H1047R, E545K, E542K, N345K, and H1047L) represented $\sim 70 \%$ of all known types of PIK3CA mutations in the dataset. Second, the therascreen PIK3CA mutational panel would represent $72 \%$ of all the known PIK3CA mutations and $80 \%$ of all patients with a known PIK3CA mutation. Third, $83 \%$ of patients with 2 or more PIK3CA known mutations would have mutations found in the therascreen panel; however, in $78 \%$ of the cases, only 1 single PIK3CA mutation would be represented in the therascreen assay. Finally, the proportion of PIK3CA mutations differed by BC subtype with $\mathrm{HR}+/ \mathrm{HER} 2-$ disease having the highest proportion, followed by HER2+ disease and TNBC. Although less frequent in the HER2+ and TNBC, the proportion is not negligible and several studies, including pivotal or registrational clinical trials, are focusing on these two populations [5-7]. To our knowledge, this is the first report to perform a comprehensive analysis of PIK3CA mutations in $\mathrm{BC}$ and to relate these findings with the type of mutations captured by the therascreen PIK3CA assay across the three main subtypes of $\mathrm{BC}$.
The SOLAR-1 phase III trial that led to the approval of alpelisib used the therascreen PIK3CA 11-mutation assay in tumor tissue to identify PIK3CA mutations [10]. From a total of 1173 patients tested for PIK3CA mutation status that had interpretable results, 341 (29\%) patients had PIK3CAmut disease [10], a proportion which is very similar $(28 \%)$ to our predicted results if the assay would have been performed in our combined dataset. More importantly, mutations in exon 9 versus exon 20 predicted similarly the degree of benefit to alpelisib in SOLAR-1 [10]. Thus, based on these results, the FDA approved the use of this assay in tumor and plasma samples as a companion diagnostic to indicate alpelisib. The approval of therascreen in plasma samples is based on a subanalysis of the SOLAR-1 trial which showed that PIK3CA mutations identified in plasma samples were also associated with treatment benefit [28].

Our results have important considerations for patients and physicians. In certain parts of the world, determination of PIK3CA status is commonplace using various types of sequencing-based assays. Some of these widely used assays such as Foundation One CDx or Guardant360 cover most or all exons of the PIK3CA gene. Thus, it is highly likely that mutations which are not part of the therascreen PIK3CA 11-mutation assay will be identified with other assays and treatment decisions will be made. In other parts of the world that have not yet implemented somatic genetic testing in $\mathrm{BC}$, the fact 
that the therascreen panel misses $\sim 20-30 \%$ of patients with known PIK3CA mutations might be a reason to choose more comprehensive PIK3CA panels.

Critical questions raised by our results are if patients with PIK3CA mutations which are not part of the therascreen panel, or hotspot and non-hotspot PIK3CA mutations identified using sequencing-based assays with higher sensitivities than therascreen, will benefit from alpelisib. For example, mutation $\mathrm{N} 345 \mathrm{~K}$ represented $5.5 \%$ of all PIK3CA mutations in the analyzed dataset and is not captured by the therascreen assay. This mutation was the fourth most frequent PIK3CA mutation in the $\mathrm{BC}$ dataset, and COSMIC [29] and OncoKB [30] datasets consider it pathogenic (score 0.95 ) and oncogenic. Moreover, N345K confers a gain of function and it has shown to increase sensitivity to PI3K inhibitors in preclinical models [23, 24]. A similar situation exists for the sixth most frequently observed PIK3CA mutation, E726K, although OncoKB [30] states that there is conflicting and/or weak data describing the oncogenic function of this mutation, it has been shown that as a single mutation it is weakly activating but as a double mutation (with E545K or H1047R) it is synergistically activating [31]. It is important to notice that the vast majority of E726K mutations are found precisely as double mutants in $\mathrm{BC}$ [31]. On the other hand, some less frequent mutations, as G1049R, have demonstrated strong driver activity in a mutation assessment platform. G1049R exhibited activity levels similar to the E542K variant with 20 -fold higher frequency [24]. Thus, better functional characterization of these and other non-hotspot PIK3CA mutations together with clinical evidence that predict benefit to alpelisib and other alpha-specific PI3K inhibitors is now of uttermost importance. At the end of the day, each type of PIK3CA mutation should be considered a biomarker by itself.

Another interesting observation is that $\sim 4 \%$ of all $\mathrm{BC}$, or $\sim 12 \%$ of all patients with PIK3CAmut $\mathrm{BC}$, have double PIK3CA mutations. Preclinically, double compound PIK3CA mutations result in increased PI3K activity and downstream signaling compared to single hotspot mutants in nontransformed cells and in $\mathrm{HR}+$ BC cells [31]. More importantly, these compound mutations seem to predict for increased sensitivity to PI3K alpha-specific inhibitors compared to single hotspot mutants in both preclinical models and also in selected patients with $\mathrm{BC}$ treated in early phase 1 trials [31]. According to our results, the therascreen panel would not capture well double PIK3CA mutations since only $5 \%$ of patients known to harbor 2 or more PIK3CA mutations would have mutations represented in the therascreen panel. Thus, if double mutations are confirmed to be a biomarker of ultra-high sensitivity to alpelisib, the therascreen assay might not be ideal for this purpose.
Our study has limitations worth noting. First, we did not evaluate the actual analytical concordance of the therascreen assay versus other sequencing assays. In other words, we assumed that the results of the combined dataset using various sequencing-based strategies was the gold standard and that the therascreen assay would identify $100 \%$ of all the PIK3CA-wild-type tumors as "no PIK3CA mutation detected" and $100 \%$ of all the PIK3CAmut tumors in the combined dataset as "PIK3$C A$ mut" if the type of mutation was on the therascreen mutation panel. However, the differences in the sensitive and specificity of the various sequencing assays will affect the concordance rates among them [32, 33]. According to the FDA therascreen PIK3CA assay specification sheet, the overall percent agreement between the therascreen assay and an NGS-based assay in SOLAR-1 was $94.7 \%$. Second, the next-generation sequencing assays and the methods used across the 10 studies evaluated in our study are highly heterogeneous and most are not standardized. Third, the analyzed datasets were mostly from primary tumor samples and acquisition of new PIK3CA mutations has been described in the metastatic setting in $8-10 \%$ of the cases [34]. Whether the frequency and spectrum of PIK3CA mutations would change if metastatic-only samples had been analyzed is currently unknown.

\section{Conclusion}

PIK3CA somatic mutations in $\mathrm{BC}$ are highly heterogenous, and the currently validated therascreen companion diagnostic test, which covers 11 hotspot mutations, might not capture up to $20 \%$ of patients with PIK3CA mutations. Thus, there is an urgent need to better understand if patients with PIK3CA mutations not detected by the therascreen assay, and predicted to be oncogenic and activating, can benefit from alpelisib or other PI3K inhibitors. Better functional characterization of these and other non-hotspot PIK3CA mutations together with further clinical studies in tumor and plasma samples from SOLAR-1 and other studies will help to better determine the population of patients who benefit from alpelisib or other alpha-specific PI3K inhibitors.

\section{Supplementary information}

Supplementary information accompanies this paper at https://doi.org/10. 1186/s13058-020-01284-9.

Additional file 1: Table A1. Main features of the 10 publicly available studies analyzed. Table A2. PIK3CA mutations found in plasma ctDNA (Guardant B360 assay) in a cohort of patients from Hospital Clinic of Barcelona.

\section{Acknowledgements}

This work was supported by Instituto de Salud Carlos III - PI16/00904 (to A.P.), PI19/01846 (to A.P.), Pas a Pas (to A.P.), Save the Mama (to A.P.), American Association of Cancer Research Career Development Awards for 
Translational Breast Cancer Research - Breast Cancer Research Foundation (Grant ID: 19-20-26-PRAT) (to A.P.), Fundación Mutua Madrileña (to A.P.), Breast Cancer Now (Grant ID: 2018NovPCC1294) (to A.P.), Fundación QuirónSalud (to A.P.), Fundación Científica Asociación Española Contra el Cáncer (Ayuda Postdoctoral AECC 2017) to F. B-M, PhD4MD (Ayuda Predoctoral) to N.C, European Union's Horizon 2020 and innovation programme under grant agreement No. 847912 (to A.P.), and Fundació La Marató TV3 under Grant ID 201935-30 (to A.P.).

\section{Authors' contributions}

$\mathrm{OM}-\mathrm{S}$ and AP have made substantial contributions to the conception and design of the work and acquisition, analysis, and interpretation of data. OM-S, NC, TP, BA, MV, BG, ES, FS, BC, FB-M, AR, DM, PG, ABR, AM, MM, and $A P$ have drafted the work or substantively revised it. The authors read and approved the final manuscript.

\section{Funding}

The authors received no specific funding for this work.

\section{Availability of data and materials}

The datasets analyzed during the current study are available in http:// cbioportal.org.

\section{Ethics approval and consent to participate}

The study was performed in accordance with Good Clinical Practice guidelines and the World Medical Association Declaration of Helsinki. Patients provided written informed consent. Approvals for the study protocol were obtained from independent ethics committees.

\section{Consent for publication}

Not applicable.

\section{Competing interests}

AP reports that his institution received research funding from Nanostring Technologies, Roche, and Novartis. AP reports consulting and lecture fees from Nanostring Technologies, Roche, Novartis, Pfizer, Oncolytics Biotech, Amgen, Lilly, MSD, and PUMA.

\section{Author details}

'Department of Medical Oncology, Hospital Clinic of Barcelona, Villarroel 170, 08035 Barcelona, Spain. ${ }^{2}$ Translational Genomics and Targeted Therapies in Solid Tumors, Institut D'Investigacions Biomèdiques August Pi i Sunyer (IDIBAPS), Villarroel 170, 08035 Barcelona, Spain. ${ }^{3}$ SOLTI Breast Cancer Research Group, Barcelona, Spain. ${ }^{4}$ Pathology Department, Hospital Clinic of Barcelona, Villarroel 170, Barcelona 08035, Spain. ${ }^{5}$ University of Napoles Federico II, Napoles, Italy.

\section{Received: 19 November 2019 Accepted: 24 April 2020}

\section{Published online: 13 May 2020}

\section{References}

1. Curtis C, et al. The Cancer Genome Atlas network. Nature. 2012;490(7418): $61-70$

2. Mollon L, Aguilar A, Anderson E, et al. Abstract 1207: a systematic literature review of the prevalence of PIK3CA mutations and mutation hotspots in HR+/HER2- metastatic breast cancer. Cancer Res. 2018;78:1207.

3. Goncalves MD, Hopkins BD, Cantley LC. Phosphatidylinositol 3-kinase, growth disorders, and cancer. N Engl J Med. 2018;379(21):2052-62.

4. Bosch A, Li Z, Bergamaschi A, Ellis H, Toska E, Prat A, et al. PI3K inhibition results in enhanced estrogen receptor function and dependence in hormone receptor-positive breast cancer. HHS Public Access. 2016;7(283).

5. Sharma P, Abramson VG, O'Dea A, et al. Clinical and biomarker results from phase I/II study of PI3K inhibitor BYL 719 (alpelisib) plus nab-paclitaxel in HER2-negative metastatic breast cancer. J Clin Oncol. 2018;36(15_suppl): 1018.

6. Kim PS, Korea S, Dent R, Centre NC, Im S, Korea S, et al. HHS Public Access 2018;18(10):1360-72.

7. Saura C, Bendell J, Jerusalem G, Su S, Ru Q, De Buck S, et al. Phase lb study of buparlisib plus trastuzumab in patients with HER2-positive advanced or metastatic breast cancer that has progressed on trastuzumab-based therapy. Clin Cancer Res. 2014;20(7):1935-45.
8. Fritsch C, Huang A, Chatenay-Rivauday C, Schnell C, Reddy A, Liu M, et al. Characterization of the novel and specific PI3Ka inhibitor NVP-BYL719 and development of the patient stratification strategy for clinical trials. Mol Cancer Ther. 2014;13(5):1117-29.

9. Juric D, Janku F, Rodón J, Burris HA, Mayer IA, Schuler M, et al. Alpelisib plus fulvestrant in PIK3CA-altered and PIK3CA-wild-type estrogen receptorpositive advanced breast cancer: a phase 1b clinical trial. JAMA Oncol. 2019; 5(2):1-9.

10. André F, Ciruelos E, Rubovszky G, Campone M, Loibl S, Rugo HS, et al. Alpelisib for PIK3CA-mutated, hormone receptor-positive advanced breast cancer. N Engl J Med. 2019;380(20):1929-40.

11. https://www.accessdata.fda.gov/cdrh_docs/pdf19/P190001C.pdf. Accessed 02 Oct 2019.

12. Martelotto LG, De Filippo MR, Ng CK, Natrajan R, Fuhrmann L, Cyrta J, et al. Genomic landscape of adenoid cystic carcinoma of the breast. J Pathol. 2015;237(2):179-89.

13. Ciriello G, Gatza ML, Beck AH, Wilkerson MD, Rhie SK, Pastore A, et al. Comprehensive molecular portraits of invasive lobular breast cancer. Cell. 2015:163(2):506-19.

14. Pereira B, Chin SF, Rueda OM, Vollan HKM, Provenzano E, Bardwell HA, et al. The somatic mutation profiles of 2,433 breast cancers refines their genomic and transcriptomic landscapes. Nat Commun. 2016;7:11479.

15. Razavi P, Chang MT, Xu G, Bandlamudi C, Dara S, Vasan N, et al. HHS Public Access. 2019;34(3):427-38.

16. Eirew P, Steif A, Khattra J, Ha G, Yap D, Biele J, et al. Dynamics of genomic clones in breast cancer patient xenografts at single cell resolution. Nature. 2016:518(7539):422-26.

17. Tan J, Ong CK, Lim WK, Ng CCY, Thike AA, Ng LM, et al. Genomic landscapes of breast fibroepithelial tumors. Nat Genet. 2015;47(11):1341-5.

18. Shah SP, Roth A, Goya R, Oloumi A, Ha G, Zhao Y, et al. The clonal and mutational evolution spectrum of primary triple-negative breast cancers. Nature. 2012:486(7403):395-9.

19. Banerji S, Cibulskis K, Rangel-Escareno C, Brown KK, Carter SL, Frederick AM, et al. Sequence analysis of mutations and translocations across breast cancer subtypes. Nature. 2012;486(7403):405-9.

20. Stephens PJ, Tarpey PS, Davies H, Van Loo P, Greenman C, Wedge DC, et al. The landscape of cancer genes and mutational processes in breast cancer. Nature. 2012;486(7403):400-4.

21. Lefebvre C, Bachelot T, Filleron T, Pedrero M, Campone M, Soria JC, et al. Mutational profile of metastatic breast cancers: a retrospective analysis. PLoS Med. 2016;13(12):e1002201.

22. Cerami E, Gao J, Dogrusoz U, Gross BE, Sumer SO, Aksoy BA, et al. The cBio Cancer Genomics Portal: an open platform for exploring multidimensional cancer genomics data. Cancer Discov. 2012;2(5):401-4.

23. Gymnopoulos M, Elsliger MA, Vogt PK. Rare cancer-specific mutations in PIK3CA show gain of function. Proc Natl Acad Sci U S A. 2007;104(13):556974.

24. Dogruluk T, Tsang YH, Espitia M, Chen F, Chen T, Chong Z, et al. Identification of variant-specific functions of PIK3CA by rapid phenotyping of rare mutations. Cancer Res. 2015;75(24):5341-54.

25. https://www.accessdata.fda.gov/scripts/cdrh/cfdocs/cfpma/pma.cfm?id=P1 90004, (May 2019).

26. Guardant Health. Guardant 360. http://www.guardant360.com/

27. Costa RLB, Han HS, Gradishar WJ. Targeting the PI3K/AKT/mTOR pathway in triple-negative breast cancer: a review. Breast Cancer Res Treat. 2018;169(3): 397-406.

28. Juric D, Ciruelos E, Rubovszky G, Campone M, Loibl S, Rugo H, et al. Abstract GS3-08: Alpelisib + fulvestrant for advanced breast cancer: subgroup analyses from the phase III SOLAR-1 trial. Cancer Res February. 2019;79:GS308.

29. Tate JG, Bamford S, Jubb HC, Sondka Z, Beare DM, Bindal N, et al. COSMIC the catalogue of somatic mutations in cancer. Nucleic Acids Res. 2019; 47(D1):D941-7.

30. Chakravarty D, Gao J, Phillips S, Kundra R, Zhang H, Wang J, et al. OncoKB: a precision oncology knowledge base. JCO Precis Oncol. 2017;1:1-16.

31. Vasan N, Razavi P, Johnson JL, Shao H, Shah H, Antoine A, et al. Double PIK3CA mutations in cis increase oncogenicity and sensitivity to PI3Ka inhibitors. Science. 2019:366(6466):714-23.

32. Supplee JG, Milan MSD, Lim LP, Potts KT, Sholl LM, Oxnard GR, et al. Sensitivity of next-generation sequencing assays detecting oncogenic fusions in plasma cell-free DNA. Lung Cancer. 2019;134:96-99. 
33. Torga G, Pienta KJ. Patient-paired sample congruence between 2 commercial liquid biopsy tests. JAMA Oncology. 2018;4(6):868-70

34. O'leary B, Cutts RJ, Liu Y, Hrebien S, Huang X, Fenwick K, et al. The genetic landscape and clonal evolution of breast cancer resistance to palbociclib plus fulvestrant in the PALOMA-3 trial. Cancer Discov. 2018;11:1390-403.

\section{Publisher's Note}

Springer Nature remains neutral with regard to jurisdictional claims in published maps and institutional affiliations.

Ready to submit your research? Choose BMC and benefit from:

- fast, convenient online submission

- thorough peer review by experienced researchers in your field

- rapid publication on acceptance

- support for research data, including large and complex data types

- gold Open Access which fosters wider collaboration and increased citations

- maximum visibility for your research: over $100 \mathrm{M}$ website views per year

At $B M C$, research is always in progress.

Learn more biomedcentral.com/submissions 